\title{
Dynamiques des systèmes \\ de production laitière, risques et transformations socio-économiques au Mali
}

\author{
B. Bonfoh ${ }^{1,2 *}$ G. Fokou ${ }^{2,3}$ M. Ould Taleb ${ }^{4}$ A. Fané ${ }^{5}$ \\ D. Woirin ${ }^{6}$ N. Laimaibao ${ }^{3}$ J. Zinsstag ${ }^{2}$
}

\begin{abstract}
Mots-clés
Production laitière - Développement agricole - Qualité - Santé - Mali.
\end{abstract}

\begin{abstract}
Résumé
En réponse à la demande laitière urbaine sans cesse croissante et aux politiques de développement du secteur, le bassin laitier situé en périphérie de Bamako a subi d'importants changements depuis l'Indépendance. Les politiques industrielles des années 1970-80 ont eu des impacts sociaux importants. Elles ont notamment conduit au développement des importations de poudre de lait et au salariat des bergers. Les politiques libérales qui ont suivi ont plutôt encouragé le développement de petites unités de transformation et l'intensification de l'élevage pastoral. Mais en l'absence de contrôle, ces politiques ont aussi contribué à d'importants mélanges génétiques, et à l'émergence de zoonoses et de risques d'infections, dangereux pour la santé publique. Une approche intégrée, liant l'adaptation des techniques de production et I'accès aux marchés et aux services, permettrait, avec l'appui des politiques et en concertation avec les acteurs, d'atténuer les risques de ces innovations et d'améliorer les moyens de subsistances de tous les acteurs.
\end{abstract}

\section{INTRODUCTION}

Au Sahel, la demande en lait et en produits laitiers est satisfaite par une offre diversifiée issue des systèmes de production locaux et des importations $(3,4)$. Les divers producteurs et transformateurs mettent à la disposition des consommateurs une gamme variée de produits qui impliquent la participation d'un nombre élevé d'intermédiaires au sein des filières (28). Le marché informel du lait est de loin le plus important et devrait contribuer à satisfaire la demande en lait et produits laitiers dans les décennies à venir $(23,46)$. Dans ce contexte, et en comparaison avec d'autres régions d'Afrique, les déterminants sanitaires sont un facteur important de la compétitivité de la filière locale (46).

Le Mali illustre cette situation relativement complexe. Ce pays présente un potentiel important en termes de productions animales

1. Centre suisse de recherche scientifique, 01 BP 1303, Abidjan 01, Côte d'Ivoire. Tél. : +22506302916 ; e-mail : bassirou.bonfoh@csrs.ci

2. Public Health and Epidemiology, Swiss Tropical Institute, Basel, Switzerland.

3. Institut du Sahel, Bamako, Mali.

4. Institut national de recherche en santé publique, Nouakchott, Mauritanie.

5. Laboratoire central vétérinaire, Bamako, Mali.

6. Vétérinaire sans frontières, Berne, Suisse.

* Auteur pour la correspondance (viande, lait, cuirs et peaux). Mais l'accès aux marchés urbains pour les éleveurs ruraux est rendu difficile par l'insuffisance des infrastructures, des services, et des soutiens institutionnels et financiers à la filière (3). Malgré un potentiel laitier significatif dû à l'importance du cheptel bovin, le pays est resté un importateur net de produits laitiers. En 2000, le lait était le troisième produit agroalimentaire le plus importé au Mali avec près de 15,5 milliards de francs CFA (30), ce qui a contribué à creuser le déficit de la balance commerciale du Mali. Les produits importés étaient consommés à plus de 90 p. 100 dans les centres urbains (22).

Les zones périurbaines des villes africaines ont subi des transformations structurelles de leur agriculture du fait d'une urbanisation rapide provoquant une forte demande en produits d'origine animale (27, 32, 33). Pour répondre à cette demande sans cesse croissante, les acteurs de la filière laitière, en l'absence de politique clairement définie, ont adopté de nouvelles stratégies et des innovations techniques $(1,2)$. Dans cette dynamique, on observe une intensification des systèmes d'élevage, un développement du secteur laitier informel, et des changements des modes de consommation $(5,29,38)$. Certaines de ces évolutions aboutissent à mettre sur le marché des produits de qualités très diverses, échappant pour la plupart à des contrôles des services publics. De fait, les normes proposées par les services techniques de l'élevage apparaissent " copiées » sur les standards internationaux et sont rarement adaptées aux conditions locales $(31,35)$. 
Le but du présent article a été de décrire l'évolution récente des politiques sectorielles mises en œuvre pour la production laitière, d'identifier les contraintes des systèmes de production et les innovations en cours, et d'évaluer les impacts de ces changements sur les acteurs, la santé des consommateurs et l'environnement de production.

\section{- METHODOLOGIE}

L'article est issu d'une synthèse bibliographique et d'observations réalisées au Mali avec le programme Lait sain pour le Sahel entre 2000 et 2006 (5, 7 à 17). Lait sain pour le Sahel a eu comme objectif d'énumérer les principaux déterminants du développement de la filière laitière locale au Sahel et de proposer les moyens d'améliorer la compétitivité des produits pour assurer les moyens de subsistance des acteurs impliqués. La filière a été analysée de manière à inscrire les facteurs de risques socio-économiques et sanitaires. Les systèmes et les techniques de production, les systèmes de collecte et de distribution, et l'évaluation des risques ont été les principales composantes de l'étude. Les informations sur les systèmes d'élevage ont été obtenues à l'aide d'enquêtes dans 198 exploitations autour de Bamako. Puis, 35 exploitations mixtes, situées à $40 \mathrm{~km}$ de Bamako et fournissant du lait à une minilaiterie gérée par une coopérative, ont été suivies. Dans ce cadre près de 301 échantillons de lait frais et fermentés on été prélevés dans les élevages et sur 15 points de vente à Bamako pour évaluer les qualités physico-chimiques et bactériologiques du lait et des produits laitiers, et déterminer à travers des études les risques pour les consommateurs. Les méthodes d'investigation ont été décrites dans le cadre du projet de politiques laitières (15). Une méthode d'amélioration de la production et de la qualité, basée sur la réduction de la contamination avec le nombre restreint des ustensiles en contact avec le lait, le lavage à l'eau propre et la désinfection à l'eau de Javel des ustensiles, a été appliquée en concertation avec les acteurs (16). Des entretiens avec certains acteurs des filières ont été conduits pour retracer les stratégies de réponse aux changements de politiques sectorielles et les attitudes face aux opportunités d'investissement et d'accès au marché. Une approche multi et transdisciplinaire a permis de décrire les transformations des systèmes de production, ainsi que les innovations technologiques et les nouvelles connaissances acquises avec des interventions pilotes. Les impacts sociaux, économiques et sanitaires combinés de ces interventions sont évalués en vue d'apporter des changements et de proposer des stratégies de production et de collecte de lait.

\section{RESULTATS ET DISCUSSION}

\section{Histoire des politiques laitières au Mali}

Depuis son indépendance, le Mali a utilisé plusieurs instruments politiques pour répondre à la problématique du développement de la filière laitière. Pour augmenter les revenus laitiers et améliorer les conditions de vie des producteurs, la politique sectorielle a eu pour objectifs jusqu'en 1985 d'intensifier les systèmes de production (amélioration de la santé, des pâturages, de la génétique) et de moderniser les circuits de commercialisation (maîtrise de la collecte, de la transformation et de la conservation).

Au début des années 1960, un comité consultatif du gouvernement avait été créé pour la gestion d'un ranch laitier. Ce ranch avait une double fonction de recherche et de production laitière à partir de bovins laitiers issus du croisement entre les races locales et exotiques $(3,4,6)$. Un plan triennal de développement économique et social (1966-68) fut élaboré en vue de l'amélioration et du développement de la production laitière au Mali. Ce plan aboutit à l'installation en 1969 de l'Union laitière de Bamako (ULB) avec le concours de l'Organisation des Nations unies pour l'enfance (Unicef) et du Programme alimentaire mondial (PAM). L'usine devait fonctionner à partir du lait local. L'utilisation des produits financiers de la vente du lait après traitement ou transformation devaient répondre aux objectifs de répartition suivants : 30 p. 100 étaient destinés à couvrir les frais de l'usine et les marges bénéficiaires de l'ULB, tandis que les 70 p. 100 restant devaient être placés dans un «Fonds de contrepartie du lait », destiné à être investi dans les exploitations laitières en vue de promouvoir la production locale $(24,49)$. Pendant cette période, les tarifs douaniers à l'importation étaient assez élevés. Considérés comme des produits de luxe, le beurre et le fromage étaient taxés à près de 86,25 p. 100, tandis que le lait en poudre était taxé à 16,1 p. 100. Cette politique permettait de générer quelques recettes douanières. Mais le faible niveau de taxation de la poudre de lait eut surtout pour effet d'encourager les importations de matière première.

Entre 1970 et 1983, l'ULB était tenue d'acheter le lait à un prix élevé afin de soutenir la production, tout en le revendant après traitement à un prix accessible aux consommateurs urbains. Cette politique correspondait à une volonté de subventionner les prix à la production et à la consommation par l'intermédiaire d'un appui budgétaire à l'ULB. Mais cette politique d'administration des prix imposée à l'ULB rendait la gestion de cette industrie délicate.

Très rapidement, alors que l'objectif recherché par les politiques était d'accroître la production locale et que l'ULB devait fournir une assistance technique aux éleveurs, les responsables se heurtèrent à une insuffisance de la collecte. Les subventions s'avérèrent coûteuses et compromirent l'objectif d'accroissement à grande échelle de la production locale. En fait, les signes d'augmentation de la production de lait local provinrent davantage des exploitations laitières périurbaines tenues par de hauts responsables politiques du pays que des exploitations paysannes familiales rurales représentant la grande majorité des producteurs.

En 1983, l'analyse des performances de l'industrie laitière de Bamako, principal outil de la politique du développement laitier au Mali, fit apparaître une évolution du secteur laitier contraire aux objectifs visés par ce modèle industriel. L'accroissement de la quantité de lait importé et transformé allait de pair avec une diminution tout aussi importante de la part du lait local dans les produits finis obtenus par les industries $(40,41)$. Le renversement du régime au pouvoir en mars 1991 contribua à la destruction des appareils productifs et montra la fragilité de la stratégie et de la politique laitière nationale.

Les réformes économiques entreprises par le gouvernement à partir de 1990 aboutirent à la remise en cause des stratégies antérieures. Le secteur laitier fut libéralisé, ce qui se traduisit par la suppression de la Société malienne des importations et des exportations (Somiex) et par la privatisation de l'ULB en 1994. Malheureusement, plus de deux décennies après l'adoption de cette politique, la production laitière continue de souffrir de la mauvaise organisation de la filière. Le contexte actuel nécessite de redéfinir la politique laitière afin de l'adapter aux changements intervenus ou en cours $(24,60)$.

\section{Autres éléments de changements structurels et conjoncturels}

La libéralisation du secteur de l'élevage et la dévaluation du franc CFA entraînèrent aussi des bouleversements dans la filière laitière $(22,25,33,47)$. Les réformes libérales eurent notamment pour effet de susciter l'émergence, à côté des industries d'Etat, de nouvelles entreprises privées (centres de collecte, collecteurs - vendeurs, minilaiteries) qui firent le lien entre les zones de production et les zones de consommation de lait (4). Mais l'approvisionnement des 
centres urbains en lait est resté un problème dans un contexte de faible production locale et de saisonnalité de la production (28).

De plus, l'urbanisation de la ville de Bamako a considérablement diminué les espaces réservés aux activités agropastorales avec, pour conséquence, l'augmentation de la distance entre les zones de production et les zones de consommation atteignant parfois plus de 100 kilomètres. L'accès au marché des produits laitiers a donc été rendu difficile et a nécessité des intermédiaires prêts à parcourir ces distances avec des risques de pertes importants (5). Malgré toutes ces contraintes et les conditions de production non incitatives, les systèmes de production locaux contribuent à l'approvisionnement de la ville de Bamako pour près de 10 p. 100 des besoins $(10,20)$.

\section{Transformation des systèmes de production laitière}

\section{Objectifs et fonctions de production des élevages laitiers}

Les fonctions de l'élevage, telles que perçues par les sociétés pastorales, évoluent en fonction des déterminants écologiques, institutionnels et économiques, d'une part, et des objectifs de production des éleveurs, d'autre part. L'élevage connaît ainsi des évolutions complexes en Afrique, liées à des dynamiques de diversification et d'intensification des systèmes de production (figure 1).

Autour de Bamako, les éleveurs ont des objectifs multiples du fait de l'existence d'un cheptel non spécialisé dans la production laitière. En 2002, seulement près de 18 p. 100 des vaches produisaient du lait (tableau I) (5). Cette proportion a été estimée à 25 p. 100 des effectifs du troupeau en 2006 (50). Suivant le niveau d'utilisation des intrants commerciaux, plusieurs systèmes de production coexistent dans cette zone et sont mis en œuvre par des éleveurs appartenant à différentes couches socioprofessionnelles. Près d'un quart (26 p. 100) des producteurs laitiers des environs de Bamako ne résident pas sur le lieu d'élevage des animaux (50). Il faut aussi noter la faible productivité des vaches laitières de races locales.

\section{Transformations sociales dans le secteur élevage en zone périurbaine}

Le processus d'intensification et de sédentarisation des sociétés pastorales peut parfois être lié à une marginalisation, à un appauvrissement, voire à une «prolétarisation» des éleveurs (18). Les

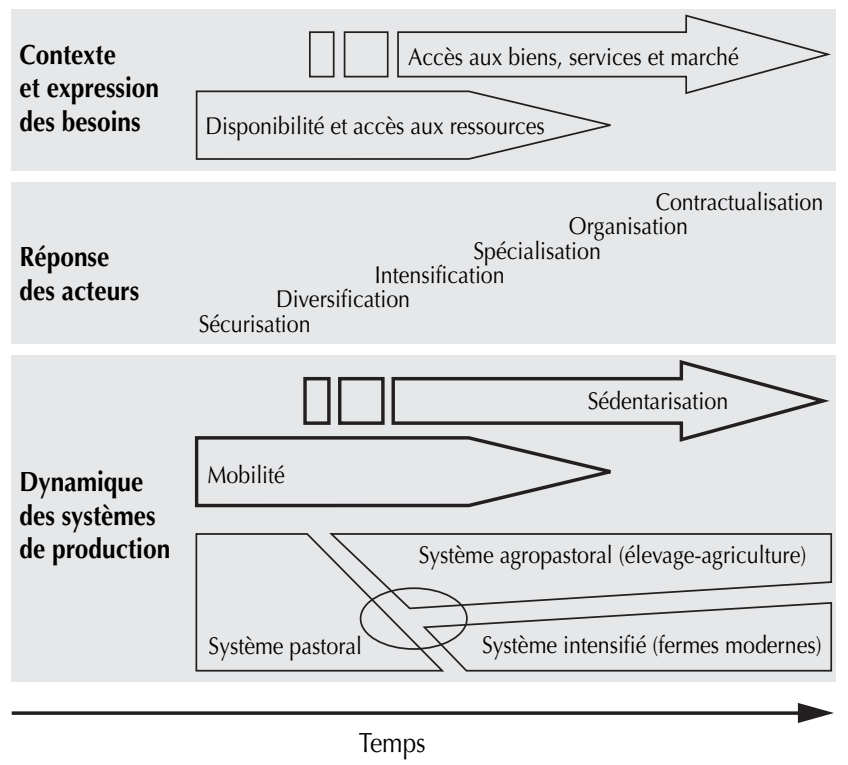

Figure 1 : transformation des systèmes de production animale dans le temps en Afrique (adapté de Djamen et coll., 2005). groupes ayant perdu leur troupeau ou ne disposant plus de bétail sont forcés de chercher du travail rémunéré dans les centres urbains (gardien de nuit, bergers, vendeurs). De telles transformations ont été décrites chez les Peuls du Mali (21). Un transfert de propriété du bétail peut notamment survenir suite à des grands changements (sécheresse et migration, développement agricole), à la fois dans les zones agricoles et dans les zones périurbaines. En effet, sous l'effet des retombées économiques de l'agriculture (par exemple, la production de coton) et de l'amélioration des revenus en milieu urbain (par exemple, liés au commerce ou aux emplois de fonctionnaires), la propriété du bétail en milieu périurbain se généralise à toutes les couches socioprofessionnelles et n'est plus du seul ressort des pasteurs.

En périphérie de Bamako sont ainsi apparus des néo-éleveurs (appelés parfois «fermiers du dimanche ») qui aménagent des petits ranchs d'agrément éloignés de leur lieu de résidence, tenus par une main d'œuvre de gardiennage bon marché. Ces couches socioprofessionnelles disposant de hauts revenus (commerçants, fonctionnaires, retraités) investissent dans l'élevage et contribuent ainsi de manière significative à l'approvisionnement des villes en lait. Par ailleurs, ces propriétaires ne disposent que de peu de temps et de savoir-faire pour apporter aux animaux tous les soins nécessaires, d'où le recours à des professionnels de l'élevage, comme le sont les bergers salariés. Les connaissances des troupeaux et de l'environnement, et le savoir-faire de ces derniers leur permettent d'être relativement bien qualifiés pour gérer les exploitations des producteurs nouvellement reconvertis à l'élevage. Le berger se voit ainsi investi de nouvelles responsabilités de gestion des troupeaux (gardiennage, stock d'aliments). Il doit aussi acheminer la production laitière jusqu'à une unité de transformation ou jusqu'au marché.

L'émergence d'une collecte régulière et sécurisée, liée à l'émergence de centres de collecte et de minilaiteries, encourage le développement de cet élevage orienté vers le marché. La livraison régulière de la production de lait à la laiterie permet à ces propriétaires de payer non seulement le salaire du berger mais également d'acheter les compléments alimentaires nécessaires au maintien de la production dans le temps.

\section{Tableau I}

Structure du cheptel bovin dans 198 exploitations dans la zone périurbaine de Bamako

\begin{tabular}{llrc} 
Classe d'âge & Age/sexe & Effectif & $\begin{array}{c}\text { Fréquence } \\
(\%)\end{array}$ \\
\hline Reproducteurs & Vaches en lactation & 1383 & 18 \\
( $\geq 4$ ans) & Vaches taries & 1516 & 20 \\
& Taureaux & 779 & 10 \\
& Sous-total & 3678 & 48 \\
Jeunes de & Taurillons & 1193 & 16 \\
remplacement & Génisses & 1204 & 16 \\
(1-3 ans) & Sous-total & 2397 & 32 \\
Veaux & Mâles & 796 & 10 \\
( $\leq 1$ an) & Femelles & 774 & 10 \\
& Sous-total & 1570 & 20 \\
Effectif total & & 7645 & 100
\end{tabular}

Source : Sall, 2002 
La responsabilisation du berger dans les fermes ne s'accompagne pas toujours d'un renforcement de ses droits historiques sur le lait. Il est souvent privé du droit de jouir de la production laitière et reçoit à la place un salaire à taux fixe très faible, réduisant ainsi sa motivation dans la bonne gestion de l'exploitation. Avec l'abandon du système de redistribution par prêt de bétail, par don de génisse ou par cession du lait de traite (55), on assiste à la mise en place d'un mode de salariat marqué par des dons d'une autre nature (habits, aliments...). Les bergers salariés sont en général très faiblement rémunérés : 51 p. 100 ont un revenu monétaire moyen variant entre 7500 à $12000 \mathrm{Fcfa}$, soit de 11,5 à $19 € /$ mois (48).

Des tensions sont fréquemment observées entre propriétaires et bergers autour de la gestion des intrants (stock d'aliments) et du lait (volume réel de lait trait). Pour les premiers, les revenus du lait ne permettent pas de couvrir les charges d'exploitation et, pour les seconds, le salaire ne couvre pas les besoins de leur famille. Par ailleurs, les activités des bergers se rendant régulièrement en ville évoluent vers d'autres types d'activités plus rémunératrices, comme la vente de paille, de volaille, ou de cartes téléphoniques). De ce fait, d'autres ethnies (Bambara, Dogon, par exemple) proposent leurs services aux propriétaires comme bergers ou contremaîtres.

Les changements de propriété du bétail en milieu périurbain et l'émergence du statut de berger salarié ont des conséquences importantes sur les systèmes techniques de production. D'une part, on assiste à un déclin dans les soins et la conduite du troupeau et, d'autre part, on note des difficultés de la part des projets de développement de la production laitière pour prendre en compte les réalités sociales complexes de l'élevage périurbain. Ces difficultés se manifestent lors de conflits fréquents dont font l'objet les livraisons de lait aux laiteries : défaut de paiement, lait de mauvaise qualité, etc.

\section{Intensification de la production laitière en zone périurbaine}

Les producteurs situés sur un rayon de 100 kilomètres autour de Bamako présentent des niveaux d'intensification très variés, allant du système pastoral extensif à l'élevage intensif en stabulation. Plusieurs d'entre eux sont membres d'une coopérative à travers laquelle ils s'approvisionnent en intrants (aliments, médicaments, géniteurs). Ceux qui pratiquent les élevages les plus intensifiés élèvent leurs animaux en régime sédentaire avec des petits déplacements en hivernage.

En moyenne, les éleveurs de la zone ont un troupeau de 52 têtes. La production moyenne de lait frais est de $1,9 \mathrm{~L}$ par vache laitière par jour avec des extrêmes compris entre 0,5 et $12 \mathrm{~L} /$ vache/jour $(5,48)$. Le volume de lait par exploitation est très variable et les plus grands producteurs produisent jusqu'à 190 litres de lait par jour, contre un litre de lait pour les plus petits (22). Le volume est proportionnel à l'effectif des vaches laitières, avec toutefois des exceptions marquées par des pics dans les élevages intensifiés par l'amélioration génétique et la complémentation alimentaire (figure 2). En milieu périurbain, 64 p. 100 des exploitations comprennent des zébus Maures, 60 p. 100 des Peuls et 56 p. 100 des Ndama. Ce sont, de loin, les trois races principales utilisées pour la production laitière (tableau II).

L'intensification des systèmes de production extensifs pour augmenter leur contribution à l'approvisionnement urbain a constitué le cœur de nombreux projets de développement. Dans cette optique, des essais de croisement entre zébus et taurins N'Dama, et entre zébus et vaches laitières de race exotique ont été effectués en station. L'option retenue a été le croisement et la sélection sur les métis pour fixer un métissage au cinq huitième. Mais les besoins alimentaires et sanitaires des animaux métis sont élevés, ce qui suppose un bon niveau de maîtrise technique. La production autour de Bamako varie entre 0,5 et $3,5 \mathrm{~L} /$ vache/jour dans les systèmes extensifs, et entre 5 et $12 \mathrm{~L} / v a c h e / j o u r$ dans les systèmes améliorés avec la complémentation alimentaire et l'amélioration génétique (5).

L'intensification de la production laitière a démarré au Mali avec un vaste programme d'insémination artificielle (IA) prenant en compte des races exotiques (Montbéliarde, Holstein, Rouge des Steppes). Aujourd'hui, les produits des principales races exotiques se retrouvent sur le terrain en milieu paysan $(3,4,22)$. Cependant, très vite l'engouement pour l'IA a fait place à un système d'utilisation de géniteurs un demi ou un quart de sang dans les exploitations. L'IA est pratiquée aujourd'hui par près de 2 p. 100 des exploitations. En revanche, l'amélioration génétique (croisement par monte naturelle) est passée de 8,3 p. 100 en 2002 à près de

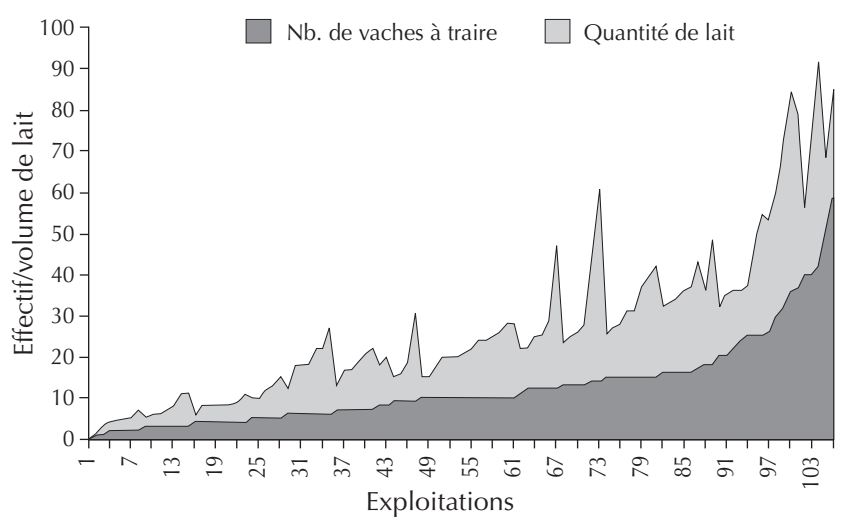

Figure 2 : relation entre l'effectif des vaches et le volume de lait produit autour de Bamako (source : Sall, 2002).

\section{Tableau II}

Fréquence des races et proportion des métisses dans les exploitations laitières autour de Bamako en 2002

\begin{tabular}{|c|c|}
\hline Race exploitée & $\begin{array}{c}\text { Fréquence dans } \\
\text { les exploitations (\%) }\end{array}$ \\
\hline \multicolumn{2}{|l|}{$\begin{array}{l}\text { Fréquence des races } \\
\text { dans les exploitations }\end{array}$} \\
\hline Zebu Maure & 64 \\
\hline Ndama & 56 \\
\hline Méré (zébu x Ndama) & 5 \\
\hline Zebu Peul & 60 \\
\hline Zébu Azawak & 6 \\
\hline Zébu Goudali & 12 \\
\hline Zébu Mbororo & 6 \\
\hline Zébu Koury & 1 \\
\hline Métis & 21 \\
\hline \multicolumn{2}{|c|}{$\begin{array}{l}\text { Proportion de sang exotique } \\
\text { chez les populations métissées ( } 21 \%)\end{array}$} \\
\hline Zébu Holstein & 4 \\
\hline Zébu Montbéliard & 13 \\
\hline Zébu Rouge des steppes & 4 \\
\hline Zébu Charolais & 1 \\
\hline Inconnu & 78 \\
\hline
\end{tabular}

Source : Sall, 2002 
41,7 p. 100 des exploitants en 2006 autour de Bamako. La pratique de l'IA n'est plus régulière à cause de son coût élevé et de l'échec des services offerts par les promoteurs locaux. En effet, l'Etat semble toujours contrôler la filière des semences dont la qualité est rarement assurée. On peut ajouter à cela la dispersion des élevages et la faible implication des organisations d'éleveurs autour de la pratique de l'IA. De ce fait, près de 78 p. 100 des métis ne sont pas caractérisés (tableau II). Sur le plan zootechnique, l'amélioration génétique permet certes de doubler le potentiel laitier, mais en l'absence de cahiers de données sur les troupeaux, il n'est pas possible de contrôler les gènes qui circulent et le risque d'érosion génétique des races autochtones est présent.

\section{Importance de l'alimentation pour l'amélioration de la production laitière}

Dans les systèmes laitiers périurbains du Mali, la majorité des producteurs affirment que l'augmentation de la production laitière n'est possible qu'avec la complémentation alimentaire des vaches laitières à base de tourteau de coton. Les suivis d'élevage montrent que ce produit, bien qu'étant cher $(0,15 € / \mathrm{kg})$, représente largement le complément le plus utilisé (tableau III). En plus des maigres pâturages, les autres aliments (paille de brousse, son de riz, sel, fanes de niébé et d'arachide) sont utilisés en appoint. Dans ces systèmes à base de tourteau, l'alimentation des vaches est très riche en protéines et relativement pauvre en énergie. Les résultats indiquent qu'en cas de déficit alimentaire, les vaches en lactation puisent dans leurs réserves corporelles pour assurer la fonction de production (17).

Les coûts de production du lait augmentent avec le niveau d'intensification, et la rentabilité de l'exploitation dépend en partie du type de complément alimentaire utilisé et de la main d'œuvre des bergers. En milieu périurbain, l'élevage laitier intensif est moins rentable que l'élevage semi-intensif ou extensif $(5,22,48)$. Cela est dû au fait qu'aucun coût n'est affecté aux pâturages et la gratuité du fourrage ingéré sur ces pâturages est le principal facteur qui explique la meilleure rentabilité des systèmes extensifs et semiintensifs.

L'influence de l'alimentation sur la fertilité d'un troupeau de vaches laitières a été estimée a entre 25 et 50 p. 100. Ainsi, l'amélioration

\section{Tableau III}

Proportion d'aliments utilisés en complément dans les exploitations laitières autour de Bamako en 2002

\begin{tabular}{|c|c|c|c|}
\hline Classe & $\begin{array}{l}\text { Type d'aliment } \\
\text { utilisé }\end{array}$ & $\begin{array}{l}\text { Nb. F } \\
\text { ploitations }\end{array}$ & $\begin{array}{l}\text { Fréquence } \\
\text { s }(\%)\end{array}$ \\
\hline \multirow[t]{2}{*}{ Fourrages } & Paille de brousse & 55 & 63 \\
\hline & Bourgou & 1 & 1 \\
\hline \multirow{3}{*}{$\begin{array}{l}\text { Résidus } \\
\text { de récolte }\end{array}$} & Fane (niébé, arachide) & 23 & 26 \\
\hline & Farine de néré & 12 & 14 \\
\hline & Graine de coton & 1 & 1 \\
\hline \multirow{3}{*}{$\begin{array}{l}\text { Sous-produits } \\
\text { agro-industriels }\end{array}$} & Tourteau de coton & 72 & 82 \\
\hline & Son (mil, riz) & 53 & 60 \\
\hline & Aliment bétail industriel & 8 & 9 \\
\hline \multirow{3}{*}{$\begin{array}{l}\text { Minéraux/ } \\
\text { vitamines }\end{array}$} & Sel gemme & 76 & 86 \\
\hline & Pierre à lécher & 5 & 6 \\
\hline & Prémix vitamines & 1 & 1 \\
\hline
\end{tabular}

Source : Sall, 2002 de l'alimentation dans les élevages intensifiés permet de plus en plus fréquemment aux vaches de mettre bas tous les 12-14 mois.

Malgré l'amélioration de la production laitière, l'intensification de la production, telle qu'elle est pratiquée en zone périurbaine, entraîne des coûts de production élevés, des problèmes sanitaires et une mauvaise gestion des ressources génétiques. L'adaptation des facteurs de production a permis, à travers une simulation obtenue auprès de 198 exploitations (figure 3), de doubler la production laitière $(5,13)$. La rentabilité d'un tel scénario est fonction de la bonne maîtrise des coûts des facteurs de production et du prix du lait au producteur.

\section{Performances et viabilité des systèmes de production et de collecte de lait}

\section{Transformation des systèmes de collecte de lait}

Les collecteurs ambulants, les centres de collecte et les minilaiteries sont aujourd'hui les principaux acteurs de la commercialisation du lait vers les centres urbains $(10,20)$. Ils remplacent petit à petit les femmes de pasteurs dans le transport du lait en direction des consommateurs, des unités de transformations et des kiosques de revente.

Le suivi de la production laitière dans 35 exploitations qui livraient le lait à une minilaiterie a montré que le niveau de la collecte avait triplé en trois ans, passant de 8000 à $30000 \mathrm{~L} /$ mois (figure 4). Les centres de collecte ou minilaiterie ont été identifiés comme étant des catalyseurs de la collecte ; c'est autour de ces structures que s'organisent de plus en plus les producteurs et l'économie des coopératives laitières. D'autres auteurs (43) ont montré que l'unité de transformation du lait constituait la « pierre angulaire » dans la construction de nouvelles filières laitières.

\section{Prix du lait et rentabilité de la production et de la collecte de lait}

Bien que, dans l'ensemble, la production de lait soit rentable pour tous les systèmes périurbains, il existe de grandes disparités entre les systèmes et entre les unités de production prises de
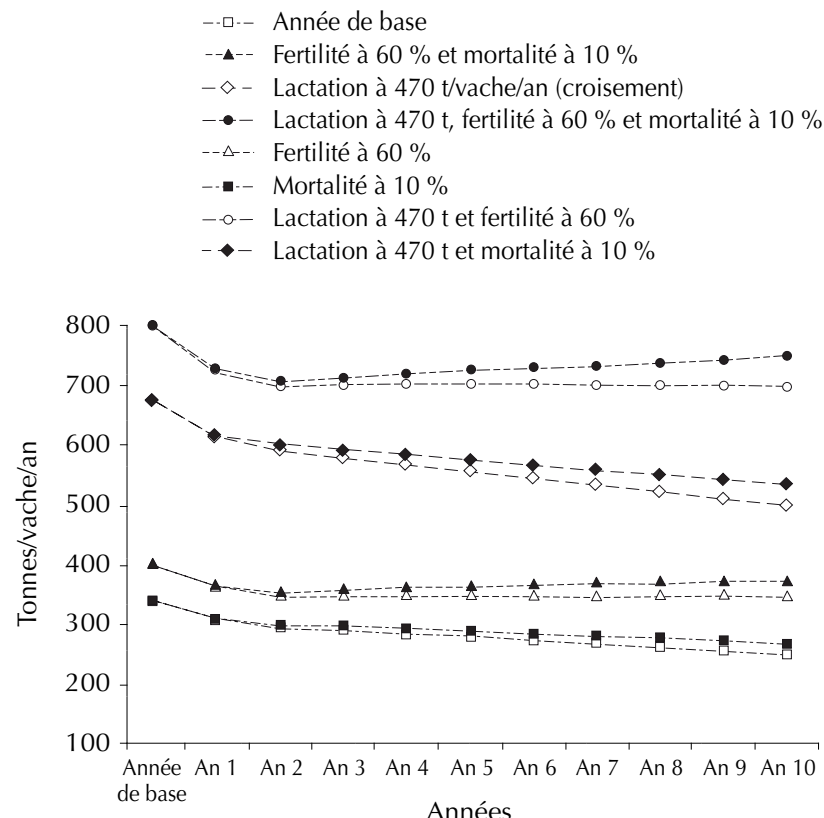

Figure 3 : simulation de la production laitière autour de Bamako en fonction des effets de modification des facteurs majeurs de production (source: Bonfoh et coll., 2006). 
façon individuelle. En particulier, les coûts de production dans les systèmes extensifs apparaissent moins élevés que dans les systèmes plus intensifiés, en raison du faible niveau des charges $(22,47)$.

En 1989-90, le lait était vendu entre 0,15 et $0,38 € / \mathrm{L}$, et le coût de production était estimé entre 0,07 et $0,23 € / L$. La marge nette variait d'une perte de $0,005 € / \mathrm{L}$ pour les concessions rurales à un profit de $0,16 \mathrm{Fcfa} / \mathrm{L}$ dans les unités villageoises (22).

En 2002, les coûts de production du litre de lait dans les élevages extensifs variaient de 0,11 à $0,16 €$, avec une marge bénéficiaire de 0,21 à $0,22 €$ à la vente (47). La complémentation représentait le plus gros poste de dépenses (48 p. 100). Les marges étaient sensiblement plus élevées en période d'abondance hivernale de lait (tableau IV).

Les statistiques de collecte et de vente de lait autour de la minilaiterie de Kasséla sont décrites dans le tableau V. Elles montrent le dynamisme de la filière locale quand les infrastructures et les

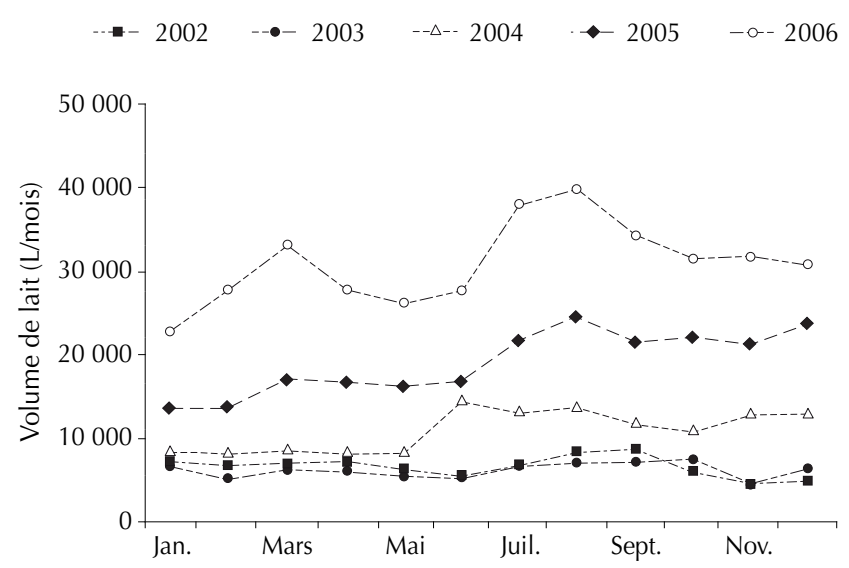

Figure 4 : variation mensuelle de la collecte de lait dans la minilaiterie de Kasséla entre 2002 et 2006 avec une intervention en juin 2004 (source : suivi de la laiterie de Kasséla par Vétérinaires sans frontières, Suisse).

\section{Tableau IV}

Evaluation des coûts de production dans les systèmes extensifs de production autour de Bamako en 2002

\begin{tabular}{|c|c|}
\hline Paramètre & Valeur \\
\hline $\mathrm{Nb}$. de laitières/unité de production & $13(18 \%)$ \\
\hline Coût variable/vache laitière/jour (Fcfa) & $0,16(100 \%)$ \\
\hline Alimentation (Fcfa) & $0,07 \quad(48 \%)$ \\
\hline Main d'œuvre (berger) (Fcfa) & $0,07 \quad(44 \%)$ \\
\hline Soins vétérinaires (Fcfa) & $0,01 \quad(8 \%)$ \\
\hline Divers (Fcfa) & $0,00(1 \%)$ \\
\hline Production de lait (L/laitière/jour) 1,10 , & $0,6-1,6$ (min. - max.) \\
\hline $\begin{array}{l}\text { Coût unitaire de production } \\
(\text { Fcfa/L) }\end{array}$ & $, 11-0,17$ (min. - max.) \\
\hline $\begin{array}{l}\text { Prix de vente du lait producteur } \\
\text { (Fcfa/L) (saison sèche }- \text { s. pluies) }\end{array}$ & $0,34-0,38$ \\
\hline $\begin{array}{l}\text { Marge bénéficiaire sur le litre produit } \\
\text { (Fcfa/L) (saison sèche - s. pluies) }\end{array}$ & $0,21-0,23$ \\
\hline
\end{tabular}

Source : Sall, 2002 services sont mis à la disposition des acteurs, respectivement sous forme de subvention et de crédit. La performance est jugée à travers trois paramètres : la marge bénéficiaire, le coût unitaire de production et la productivité (22).

\section{Frein à l'élan de production modernisée, mais développement de la filière}

Tous les circuits de production ont subi l'influence des réformes politiques et économiques. La filière a notamment été touchée par la libéralisation du secteur de l'élevage et la dévaluation du franc CFA (25). Or, les impacts de ces réformes politiques sont relativement complexes. Le développement de la production laitière locale n'a pas semblé connaître l'élan attendu. Les coûts de production ont augmenté et les importations de lait en poudre ont paradoxalement augmenté (5). Les promoteurs des fermes laitières de grande taille ne sont pas parvenus à tirer pleinement partie de ces réformes, du fait de l'absence de consensus autour du prix du litre, de l'irrégularité de la collecte et surtout des problèmes de rejet de lait non conforme aux normes de qualité de la principale industrie privée (5). Des distorsions dans la commercialisation sont apparues. Les coûts élevés de certains facteurs de production, comme l'alimentation et la faible demande pour du lait produit localement, se sont traduits par une impasse pour la filière laitière industrielle, telle qu'elle avait été imaginée dans les stratégies de développement du secteur laitier au Mali.

\section{Tableau V}

Fonctions de collecte et de distribution du lait par la laiterie de Kasséla en 2006

\begin{tabular}{|c|c|c|}
\hline Paramètre & Volume (L) & Pourcentage \\
\hline \multicolumn{3}{|l|}{ Fournisseur en lait } \\
\hline Coopérative de Koro-Koro & 17484 & 5,0 \\
\hline Coopérative de Markakoungo & 96097 & 26,0 \\
\hline Membre coopérative de Kasséla & 135611 & 36,0 \\
\hline $\begin{array}{l}\text { Non-membre coopérative } \\
\text { de Kasséla }\end{array}$ & 121935 & 33,0 \\
\hline Total & 371125 & 100 \\
\hline \multicolumn{3}{|l|}{ Distribution du lait } \\
\hline Vente au détail lait pasteurisé & 170463 & 45,9 \\
\hline Don & 693 & 0,2 \\
\hline Pertes & 5484 & 1,5 \\
\hline $\begin{array}{l}\text { Vente aux industries } \\
\text { (Eurolait \& Mali-Lait) }\end{array}$ & 148385 & 40,0 \\
\hline $\begin{array}{l}\text { Vente aux grossistes } \\
\text { (lait pasteurisé) }\end{array}$ & 46101 & 12,4 \\
\hline Total & 371125 & 100 \\
\hline \multicolumn{3}{|l|}{ Nature du lait vendu } \\
\hline Lait cru & 131093 & 36 \\
\hline Féné * & 6202 & 2 \\
\hline Lait pasteurisé & 228347 & 62 \\
\hline Total & 365641 & 100 \\
\hline
\end{tabular}

Source : suivi de la laiterie de Kasséla par Vétérinaires sans frontières, Suisse (2006) * Féné : lait pasteurisé spontanément fermenté 
Cependant, la plupart des producteurs sont devenus vendeurs directs de leurs produits à Bamako à des prix relativement élevés $(0,61-0,91 € / L)$. De nouveaux acteurs intermédiaires sont apparus dans le secteur de la collecte, de la transformation et de la distribution, et de nombreux emplois ont été créés dans le système périurbain. L'analyse des comptes de résultat de la minilaiterie de Kasséla montre, par exemple, un bénéfice de $9906 €$ /an pour l'ensemble des acteurs de la filière. La filière a donc bien connu un développement, mais sous des formes relativement inattendues.

\section{Conséquences des transformations des systèmes de production et de collecte}

Pour répondre à la demande, les transformations des systèmes de production laitière locale ont eu des répercussions importantes sur les performances financières des acteurs des filières. Elles ont cependant aussi entrainé l'émergence de pathologies nouvelles et de risques sur la santé publique.

\section{Impacts sur la santé animale}

Dans les élevages intensifiés, la mortalité des jeunes animaux est devenue fréquente. Alors que le taux de mortalité des veaux pendant la première année de vie était de 10 p. 100 en élevage traditionnel, il était de 19 p. 100 en élevage intensifié privé et de 25 p. 100 en station de recherche [hazard ratio : 2,2 ; intervalle de confiance (IC) 1,2-3,8; p = 0,01] (60). En moyenne, dans la zone périurbaine de Bamako, il était de 17 p. 100. Le taux de mortalité des veaux en zone périurbaine a donc doublé avec la modernisation de l'élevage. Cette augmentation de la mortalité des veaux a limité l'augmentation de la productivité des troupeaux.

La principale cause de mortalité en élevage traditionnel est la sousalimentation des veaux. En revanche, dans les élevages intensifiés, les principales causes de mortalité identifiées étaient les troubles digestifs parasitaires ou infectieux (28 p. 100), les pathologies périnatales (16 p. 100) et les accidents (14 p. 100) (60). Les déterminants de cette mortalité étaient l'insuffisance d'hygiène et de surveillance des troupeaux, et la quantité élevée du lait prélevé alors que les parasitoses gastro-intestinales étaient les causes majeures en élevage modernisé $(59,60)$. L'augmentation des risques de mortalité des veaux (hazard ratio : 3,5 ; IC : 1,8-6,6 ; p > 0,01) serait aussi le reflet du nombre réduit de bergers, et du manque de confiance entre ces derniers et les propriétaires. Cette relation conflictuelle rend impossible la détermination précise des coûts de production pour planifier les investissements et le développement de la production (58). L'assainissement des relations entre les bergers et les propriétaires passerait par une reconnaissance du statut des bergers, de leur cahier des charges, et enfin par la valorisation de leur rémunération en relation avec les résultats de la production.

Par ailleurs, autour de Bamako, l'intensification du système de production a augmenté de façon significative la prévalence de la brucellose dans les exploitations. Il s'agit là d'une conséquence des croisements incontrôlés et des systèmes de conduite groupée des troupeaux. Les échanges de géniteurs et la vente des reproductrices se sont développés avec l'amélioration génétique. Cette pratique a entraîné la diffusion entre troupeaux de la brucellose et d'autres pathologies. Il en résulte que la prévalence des anticorps de brucella dans le lait mélangé des exploitations était de 35 p. 100 en milieu périurbain (Bamako) et seulement de 4 p. 100 en milieu rural. Avec la brucellose, on peut observer une réduction de la fertilité des vaches de 20 p. 100 et de la production laitière de 16 p. 100 chez les séropositifs (11). Il est aussi certain que l'amélioration génétique réduit la résistance des animaux (51).

Environ 75 p. 100 des produits laitiers frais proviennent de fermes où l'on a détecté des mammites subcliniques (9). Cette pathologie augmente avec la production laitière par vache. Elle entraîne non seulement des pertes de 15 à 35 p. 100 de la production de lait, mais aussi un risque élevé d'infection à staphylocoque pour le consommateur. Lorsqu'elles sont fréquemment traitées, les mammites sont à l'origine de la présence de résidus d'antibiotiques dans le lait, si les délais d'attente ne sont pas respectés. Ainsi, le taux de prévalence des inhibiteurs était de 6 p. 100 dans le lait frais, montrant un risque chez le consommateur à Bamako (hazard ratio : 5,1 ; IC : 1,1-24,7) (7). Un taux de 50 p. 100 a même été observé pendant la même étude chez les fournisseurs d'industries laitières à Niamey.

\section{Impacts sur la qualité du lait et risques pour la santé publique}

Le lait et les produits laitiers traités, vendus et consommés au Sahel sont reconnus comme étant de qualité bactériologique et hygiénique douteuse $(8,11,14,16,19,56)$. Ils contiennent aussi bien les germes d'altération que des germes pathogènes (Staphylococcus sp., E. coli) et zoonotiques (Brucella sp.) (34). La situation de l'hygiène et de la qualité du lait dans les systèmes extensifs de production est problématique et dépend non seulement du système d'élevage mais aussi des conditions du climat, des infrastructures, de la technologie (43) et des nouvelles pratiques observées dans le secteur informel.

Quel que soit le système de production et de collecte, le lait arrivant sur le marché ou chez les consommateurs avec des charges bactériennes élevées est moins facilement valorisable. Lors du chauffage ou de la transformation, une partie du lait peut être perdue. Ce lait représente aussi un risque sanitaire (zoonoses) s'il est consommé cru. Le système de collecte est ainsi une des principales contraintes au développement du commerce du lait local (10). La résultante du manque d'hygiène dans les nouveaux systèmes de production et de collecte est l'augmentation des risques sanitaires pour les consommateurs de lait et de produits laitiers. Bien sûr ce risque peut être relativement faible en comparaison aux risques des principales maladies endémiques.

Les insuffisances et les inadaptations des instruments légaux de contrôle en cours intensifient les risques, surtout dans le secteur informel. A Bamako, 97,4 p. 100 des individus vivant en ville déclarent avoir consommé au moins une fois du lait dans les deux semaines précédentes. Le lait en poudre est le plus fréquemment utilisé. Le lait cru et le lait bouilli ou pasteurisé sont consommés tel quel, alors que le lait fermenté est consommé avec les plats chauds (bouillie) (38). Une attention particulière devrait être portée sur les produits considérés comme sains (lait chauffé, pasteurisé...) car ce sont ces produits qui causent le plus d'intoxications. D'ailleurs, la consommation régulière de lait dit bouilli (hazard ratio : 4,38; IC : 1,15-16,71; p < 0,03), le jeune âge du consommateur et la possession de latrines traditionnelles étaient les principaux facteurs de risque pour les toxi-infections alimentaires avec des symptômes de diarrhées et de vomissement. La plupart des personnes n'étaient pas conscientes de la contribution des aliments - et plus précisément du lait - dans les maladies diarrhéiques $(11,37)$.

D'autre part, au Mali, parmi les patients $(\mathrm{n}=168)$ ayant consulté deux cliniques pour des cas de fièvre, 10,1 p. 100 étaient séropositifs à la brucellose avec le test du rose bengale. Ce taux était de 18,2 p. $100 \mathrm{chez}$ les patients résidant à Bamako et de 3,3 p. 100 chez ceux résidant à Mopti. Une corrélation significative $(\mathrm{P}<0,05)$ a été montrée entre la consommation de lait cru et la séropositivité $(11,52)$. Ce taux reflète bien le niveau de prévalence de la brucellose dans les exploitations. Ce résultat pose le problème du diagnostic différentiel chez les patients et appelle à une approche intersectorielle du diagnostic des zoonoses (53). 


\section{Actions pilotes d'amélioration de la qualité et des services aux producteurs}

Une méthode d'amélioration de la production et de la qualité basée sur la réduction de la contamination a été testée par le projet « Lait sain ». Elle a permis de ramener la contamination à un niveau où il pouvait être pasteurisé sans perte, soit de $10^{7}$ à $10^{5} \mathrm{ufc} / \mathrm{ml}$. Cette méthode, qui implique la minimisation des pertes par un transport sécurisé, peut fonctionner si les coûts de l'hygiène sont réduits pour les producteurs, et si le lait est acheté à la qualité par les minilaiteries et les industries. Le comportement et la perception des producteurs autour de cette qualité restent cependant des facteurs déterminants dans l'amélioration de l'hygiène $(12,16,36)$.

Ainsi, il est important que l'amélioration de l'hygiène et de la qualité des produits apporte une plus-value monétaire concrète aux acteurs. Cette « rémunération » du bon producteur passe par un maillon essentiel qui est le consommateur ou les industries. A cet effet, la concertation entre les différents maillons de la filière s'avère nécessaire pour dynamiser la plate-forme de veille, de reconnaissance et de promotion de la qualité.

La méthode intégrée d'amélioration de la production et de la qualité reposait sur la mise en place d'un schéma fonctionnel de services aux éleveurs et l'institution d'une gestion privée des infrastructures laitières. Le mode de financement qui sous-tendait cette approche comprenait trois composantes : la subvention pour les investissements lourds, la constitution d'un fonds de garanti pour le crédit sur les postes de production, et l'appui technique dans l'organisation et la contractualisation avec les services privés et publics. Le remboursement des crédits s'effectuait par les prélèvements directs sur les ventes de lait. Le contrôle de qualité à tous les niveaux permettait ainsi d'assurer la transformation, la garantie sanitaire et surtout la gestion des litiges entre les acteurs de la chaîne. D'après la majorité des acteurs, l'adoption de la méthode reposait essentiellement sur l'accès aux crédits, l'amélioration de l'hygiène et de la qualité des produits. La figure 5 montre le cycle de la méthode de développement local de la filière.

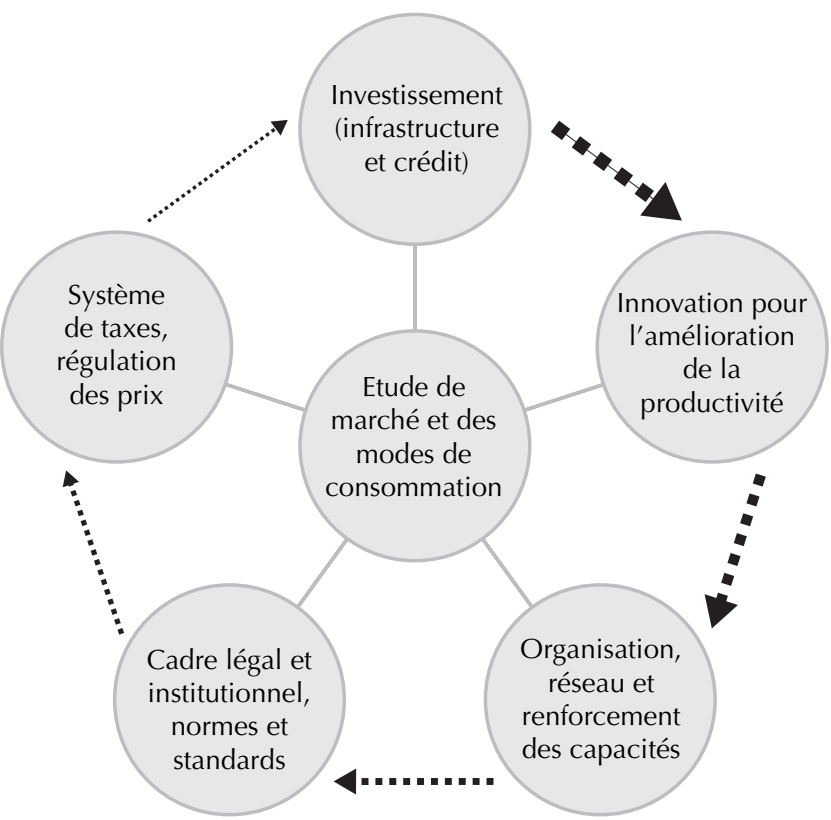

Figure 5 : Approche intégrée et cycle de développement de la filière laitière.

\section{CONCLUSION}

Le système extensif de production laitière est resté la principale activité économique agricole dans les zones urbaines sèches. Il est sous la pression des récents développements du contexte économique, social et politique. Les stratégies et les politiques laitières d'après les indépendances, qui étaient basées sur les modèles industriels, n'ont pas donné les résultats escomptés, au même titre que les récentes transformations économiques marquées par la privatisation et la dévaluation du franc CFA. Il s'est donc développé un dynamisme du secteur informel et des adaptations des acteurs aux nouvelles conditions avec des améliorations de production qui ne sont pas sans conséquences sur la santé publique. Cependant, avec les efforts engagés par les acteurs pour satisfaire une demande en lait sans cesse croissante, l'intensification de la production laitière périurbaine, couplée au secteur des importations, vont contribuer à répondre aux exigences du marché et à assurer les revenus des acteurs.

Le développement de la production laitière n'est pas seulement un problème technique et technologique. Il s'aligne aussi sur des logiques économiques, politiques et institutionnelles. Les agents recherchent, par exemple, la maîtrise des coûts de production, veulent limiter les risques de pertes et évoluent dans un cadre de compétences limitées. Ainsi, la promotion de filières laitières locales doit s'accompagner de changements dans l'accès aux infrastructures et aux services, et dans l'organisation des relations entre acteurs. Le secteur laitier a montré que les centres de collecte et les minilaiteries ont constitué des instruments performants d'organisation des filières et de mobilisation collective des acteurs $(2,27$, 28). L'organisation autour de ces infrastructures et des besoins de services devrait promouvoir l'adoption des innovations techniques pour améliorer la production et la rentabilité des systèmes de production et de collecte. L'accès à certains services clés comme le crédit, la formation en hygiène et en assurance qualité a été déterminant dans l'amélioration de la production et de la collecte du lait local (5).

Il est apparu, au cours de cette analyse synthétique, que la maittrise des coûts de production est sous-tendue par la diversification des aliments, l'amélioration de la qualité du lait et des relations entre bergers et propriétaires $(2,26,39)$. Pour ce qui concerne la qualité, une évaluation participative des risques (16) et la fixation concertée des normes (12) permettraient une adoption du contrôle de qualité comme faisant partie intégrante du système de production. Le développement de la filière locale est basé sur les fondements économiques et institutionnels à travers les infrastructures privées (subvention des centres de collecte et des minilaiteries), soutenues par des services (crédits, formation en hygiène et qualité).

\section{Remerciements}

Le présent article est basé sur les résultats du projet Lait sain pour le sahel, conduit en partenariat avec le Laboratoire central vétérinaire de Bamako, l'Institut du Sahel, l'Institut tropical suisse et l'Ecole polytechnique fédérale de Zurich. Ce projet multidisciplinaire a été conduit au Mali, au Niger et en Mauritanie. L'article a été préparé avec l'appui du Pôle de partenariat suisse de recherche Nord-Sud (Nccr-North-South) : Partenariat de recherche pour l'atténuation des syndromes du changement global. Le Nccr-NorthSouth est cofinancé par la Direction pour le développement de la coopération Suisse (DDC) et le Fond national suisse de recherche scientifique (FNS). 


\section{BIBLIOGRAPHIE}

1. BA DIAO M., SENGHOR C.D., DIAO B., THYS E., 2002. Production et transformation du lait en région agropastorale au Sénégal : cas de la zone périurbaine de Kolda. Revue Elev. Méd. vét. Pays trop., 55 : 221-228.

2. BA DIAO M., TRAORE E.H., DIENG A., SALL C., SOW O.S., TONFIO R., 2004. Petites entreprises de transformation et développement laitier dans la vallée du fleuve Sénégal. RASPA, 1 : 25-30.

3. BELLINGUEZ A., 1994. Etude de l'impact des projets sur les systèmes de production laitiers périurbains à Bamako. Mém. Ing. Tech. agric. régions chaudes, Eitarc/Cnearc, Montpellier, France, 98 p.

4. BELLINGUEZ A., 1998. Mise en place de centres de collecte de lait frais dans la zone périurbaine de Bamako. In : Actes atelier int. Marchés urbains et développement laitier en Afrique sub-saharienne, Montpellier, France, 9-10 sept. 1998. Versailles, France, Quae, p. 7.

5. BONFOH B., ANKERS P., SALL A., DIABATE M., TEMBELY S., FARAH Z., ALFAROUKH I.O., ZINSSTAG J., 2006. Schéma fonctionnel de services aux petits producteurs laitiers périurbains de Bamako (Mali). Revue Etud. Rech. sahél., 12 : 7-25.

6. BONFOH B., CORNIAUX C., COULIBALY D., DIABATE M., DIALLO A., FANE A., KONE Y., NAPO A., POCCARD CHAPUIS R., TRAORE A., 2006. Synthèse bibliographique sur les filières laitières au Mali. Dakar, Sénégal, Isra. http://www.repol.info/IMG/pdf/Synthese_biblio_du_Mali.pdf 7. BONFOH B., DEM S., KEITA O., DELORENZI S., TRAORE H., SIMBE C.F., ALFAROUKH I.O., FARAH Z., NICOLET J., ZINSSTAG J., 2003. Assessment of antibiotics residues by microbial inhibitor tests in cow's fresh milk sold in Bamako, Mali. Milk Sci. int., 58: 304-307.

8. BONFOH B., FANE A., TRAORE N.A., COULIBALY Z., SIMBE C.F., ALFAROUKH O.I., NICOLET J., FARAH Z., ZINSSTAG J., 2002. Qualité microbiologique du lait et des produits laitiers vendus en saison chaude dans le district de Bamako au Mali. Bioterre ( $\mathrm{n}^{\circ}$ spécial) : 242-250.

9. BONFOH B., FANE A., DEM S., TRAORE H., SIMBE C.F., ALFAROUKH I.O., NICOLET J., REHBERGER R., FARAH Z., ZINSSTAG J., 2003. Caractéristiques physico-chimiques et biologiques du lait et des produits laitiers vendus à Bamako. Revue Etud. Rech. sahél., 8-9 : 8-12.

10. BONFOH B., FANE A., NETOYO L., MBAYE Y., SIMBE C.F., ALFAROUKH I.O., NICOLET J., FARAH Z., ZINSSTAG J., 2003. Collecte et distribution du lait produit localement en zone périurbaine de Bamako (Mali). Revue Etud. Rech. sahél., 8-9 : 13-18.

11. BONFOH B., FANE A., STEINMANN P., HETZEL M., TRAORE A.N., TRAORE M., SIMBE C.F., ALFAROUKH I.O., NICOLET J., AKAKPO J.A., FARAH Z., ZINSSTAG J., 2003. Qualité microbiologiques du lait et des produits laitiers vendus au Mali et leurs implications en santé publique. Revue Etud. Rech. sahél., 8-9 : 19-27.

12. BONFOH B., ROTH C., TRAORE A.N., FANE A., SIMBE C.F., ALFAROUKH I.O., NICOLET J., FARAH Z., ZINSSTAG J., 2005. Effect of washing and disinfecting containers on the microbiological quality of fresh milk sold in Bamako (Mali). Food control, 17: 153-161.

13. BONFOH B., SALL A., DIABATE M., DIARRA A., NETOYO L., MBAYE Y., SIMBE C.F., ALFAROUKH O.I., FARAH Z., ZINSSTAG J., 2003. Viabilité technico-économique du système extensif de production et de collecte de lait à Bamako. Revue Etud. Rech. sahél., 8-9 : 173-184.

14. BONFOH B., WASEM A., ROTH C., FANE A., TRAORE A.N., SIMBE C.F., ALFAROUKH I.O., NICOLET J., FARAH Z., ZINSSTAG J., 2003. Les sources de contamination du lait du lait local et les méthodes d'amélioration de sa qualité microbiologique à Bamako (Mali). Revue Etud. Rech. sahél., 8-9 : 29-37.

15. BONFOH B., WASEM A., ROTH C., HETZEL M., STEINMANN P., ZINSSTAG J. L'hygiène et la qualité sanitaire du lait et des produits laitiers: implications en santé publique. Dakar, Sénégal, Isra. (Notes méthodologiques $\mathrm{n}^{\circ}$ 8) http://www.repol.info/IMG/pdf/Note_methodo_ securite_sanitaire.pdf

16. BONFOH B., WASEM A., TRAORE A.N., FANE A., SPILLMAN H. SIMBE C.F., ALFAROUKH I.O., NICOLET J., FARAH Z., ZINSSTAG J., 2002. The milk microbiological contamination chain from the cow's udder to the selling point in Bamako, Mali. Food Control, 14: 495-500.

17. BONFOH B., ZINSSTAG J., FARAH Z., SIMBE C.F., ALFAROUKH I.O., AEBI R., BADERTSCHER R., COLLOMB M., MEYER J., REHBERGER B., 2005. Raw milk composition of Malian Zebu cows (Bos indicus) raised under traditional system. Food Compos. Anal., 18: 29-38.

18. BOVIN M., MANGER L., 1989. Adaptative strategies in African arid lands. In: Proc. Semin. Scandinavian Institute of African Studies, Uppsala, Sweden.
19. CAPLICE E., FITZGERALD G.F., 1999. Food fermentations: role of micro organisms in food production and preservation. Int. J. Food Microbiol., 50: 131-149.

20. CORNIAUX C., DUTEURTRE G., DIEYE P.N., POCCARD-CHAPUIS R., 2005. Les minilaiteries comme modèle d'organisation des filières laitières en Afrique de l'Ouest : succès et limites. Revue Elev. Méd. vét. Pays trop., 58 : 237-243.

21. DE BRUIJN M., VAN DIJK H., 2003. Changing population mobility in West Africa: Fulbe pastoralists in Central and South Mali. Afr. Affairs, 102: 285-307

22. DEBRAH S., SISSOKO K., SOUMARE S., 1995. Etude économique de la production laitière dans la zone périurbaine de Bamako au Mali. Revue Elev. Méd. vét. Pays trop., 48 : 101-109.

23. DELGADO C., ROSENGRANT M., STEINFELD H., EHUI S. COURBOIS C., 1999. Livestock to 2020. The next food revolution. Washington, DC, USA, International Food Policy Research Institute, p. 1-83. (Discussion paper No 28)

24. DEMBELE N., 1995. Etude économique de la disponibilité et de I'utilisation des suppléments dans I'alimentation des bovins au Mali. Etude de cas des éleveurs du Cercle de Koutiala. Bamako, Mali, Isfra/IER, 159 p.

25. DIAGANA B., AKINDES F., SAVADOGO K., REARDON T., STAATZ J., 1999. Effects of the CFA franc devaluation on urban food consumption in West Africa: overview and cross-country comparisons. Food Policy, 24: 465 .

26. DIAO BA M., SENGHOR C.D., DIAO B., 2002. Les femmes dans la filière lait périurbaine au Sénégal. Cas de la région de Kolda. Revue Elev. Méd. vét. Pays trop., 55 : 299-304.

27. DIEYE P.N., DUTEURTRE G., SISSOKHO M.M., SALL M., DIA D. 2003. La production laitière périurbaine au sud du Sénégal. Saisonnalité de l'offre et performances économiques. Tropicultura, 21 : 142-148.

28. DIEYE P.N., FAYE A., SEYDI M., CISSE S.A., 2002. Production laitière périurbaine et amélioration des revenus des petits producteurs en milieu rural au Sénégal. Cah. Agric., 11 : 251-257.

29. DJAMEN P., LOSSOUARN J., HAVARD M., OLLIVIER B., 2005 Développement des filières et dynamique du changement : quelles perspectives pour les élevages bovins de la Vina (Cameroun). In : Symp. int. Développement des filières agropastorales en Afrique, Niamey, Niger, 21-27 fév. 2005. Wageningen, Netherland, CTA.

30. DNS, 1999. Statistiques annuelles des produits alimentaires au Mali. Bamako Mali, Direction nationale des statistiques.

31. DUTEURTRE G., 2004. Normes exogènes et traditions locales la problématique de la qualité dans les filières laitières africaines. Agricultures, 13 : 91-98.

32. DUTEURTRE G., DIEYE P.N., BONFOH B., POCCARD-CHAPUIS R., BROUTIN C., 2005. Filières laitières et territoires : les espaces agricoles de l'Uemoa face à l'ouverture des marchés. In : Symp. int. Développement des filières agropastorales en Afrique, Niamey, Niger, 21-27 fév. 2005. Wageningen, Netherland, CTA.

33. DUTEURTRE G., DIEYE P.N., DIA D., 2005. L'impact des importation de volailles et de produits laitiers sur la production locale au Sénégal. Dakar, Sénégal, Isra, p. 78. (Etudes et documents vol. 8, $\mathrm{n}^{\circ} 1$ )

34. FANE A., BONFOH B., ZINSSTAG J., FARAH Z., NIANG M. TEMBELY S., ALFAROUKH I.O., AKAKPO J.A., 2005. Effet de la fermentation du lait sur la survie de Brucella abortus. RASPA, 3 : 1-6.

35. FAO/WHO, 1997. General requirements (food hygiene). Codex Alimentarius suppl. to Vol. 1B. Rome, Italy, FAO.

36. GRILLET N., GRIMAUD P., LOISEAU G., WESUTA M., FAYE B., 2005. Qualité sanitaire du lait cru tout au long de la filière dans le district de Mbarara et la ville de Kampala en Ouganda. Revue Elev. Méd. vét. Pays trop., 58 : 245-255

37. HETZEL M.W., BONFOH B., FARAH Z., SIMBE C.F., ALFAROUKH O.I., ZINSSTAG J., 2005. Milk consumption pattern in an area with traditional milk production: data from a case control study in peri-urban Bamako, Mali. RASPA, 3: 174-177.

38. HETZEL M.W., BONFOH B., FARAH Z., TRAORE M., SIMBE C.F., ALFAROUKH I., SCHELLING E., TANNER M., ZINSSTAG J., 2004 Diarrhoea, vomiting and the role of milk consumption: perceived and identified risk in Bamako (Mali). Trop. Med. int. Health, 9: 1132-1138.

39. HURSEY B., SLINGENBERGH J., 1998. Livestock and human needs. Rome, Italy, FAO. http://www.fao.org/ag/magazine/9812int.htm

40. MDR, 1985. Politique laitière au Mali. Bamako, Mali, ministère du Développement rural. 
41. MDR, 1986. Analyse de la situation laitière. In : Journées nationales de réflexion sur le secteur Elevage, Bamako, Mali, 16-19 déc. 1986 Bamako, Mali, ministère du Développement rural.

42. MEIR R., 2006. Durabilité écologique de la production laitière en zone périurbaine de Bamako. Cas de l'élevage autour de la forêt classée de Faya à Kasséla. Rapport de stage. Zurich, Suisse, Eidgenössische Technische Hochschule, 18 p.

43. MENSAH P., 1997. Fermentation - the key to food safety assurance in Africa. Food Control, 8: 271-278.

44. METZGER R., CENTRES J.M., LAMBERT J.C. 1995. L'approvisionnement des villes africaines en lait et en produits laitiers. Paris, France, Lavoisier, 105 p. (FAO Production et santé animales $n^{\circ} 124$ ) 45. MUSTERMANN S., SOMDA J., KAMUANGA M., HEMPEN M. UNGER F., CARAYOL D., 2003. Small scale milk transformation to enhance value added milk production from local dairy sector of the periurban markets in The Gambia. Revue Etud. Rech. sahél., 8-9 : 133-139.

46. OMORE A.O., STAAL S.J., OSAFO E.L.K., KURWIJILA L., BARTON D., MDOE N., 2004. Market mechanisms, efficiency, processing and public health risks in peri-urban dairy product markets: synthesis of findings from Ghana and Tanzania. Nairobi, Kenya, ILRI, p. 1-131.

47. OWANGO M.O., STAAL J.S., KENYANJUI M., LUKUYU B., NJUB D., THORPE W., 1998. Dairy co-operatives and policy reform in Kenya: effects of livestock service and milk market liberalization. Food Policy, 23: 173-185.

48. SALL A., 2002. Evaluation de la viabilité des systèmes de production et de collecte de lait de vache dans la ceinture laitière de Bamako. Mém. Zootech., IPR/Ifra, Bamako, Mali, 61p.

49. SANDFORD S., 1983. Management of pastoral development in the Third World. John Wiley, New York, NY, USA.

50. SERY, 2006. Impacts des minilaiteries sur les transformations socioéconomiques et culturelles au Mali. Bamako, Mali, Institut du Sahel.

\section{Summary}

Bonfoh B., Fokou G., Ould Taleb M., Fané A., Woirin D., Laimaibao N., Zinsstag J. Dynamics of Dairy Production Systems, Risks, and Socio-Economic Change in Mali

In response to the ever increasing urban milk demand and to the subsector's development policies, the suburban milk basin of Bamako has changed a lot since the Independence. The industrial policies of the 1970-80's had major social impacts. They led in particular to the development of dry milk imports and to herdsmen becoming salaried employees. The ensuing liberal policies promoted the development of small processing units and pastoral farming intensification. But, without control, these policies also contributed to much genetic mixing and to the emergence of zoonoses and infection risks, representing a danger for public health. An integrated approach, linking adapted production techniques to the access to markets and services, could help - with policy support and in concert with actors - to mitigate risks from innovations and improve the livelihoods of all stakeholders.

Keywords: Milk production - Agricultural development Quality - Health - Mali.
51. SHANG D., XIAO D., YIN J., 2002. Epidemiology and control of brucellosis in China. Vet. Microbiol., 90: 165-182.

52. STEINMANN P., 2003. Brucellosis and Q-fever in Mali: Case detection, role of milk contamination and other risk factors. Thesis Biology, University of Basel, Switzerland, $77 \mathrm{p}$

53. STEINMANN P., BONFOH B., PETER O., SCHELLING E., TRAORE M., ZINSSTAG J., 2005. Seroprevalence of Q-fever in febrile individuals in Mali. Trop. Med. int. Health, 10: 612-617.

54. STOBBS T.H., THOMPSON P.A.C., 1975. Milk production from tropical pastures. World Anim. Rev., 13. http://www.fao.org/docrep/004/ x6512e/X6512E04.htm

55. THEBAUD B., 1998. Elevage et développement au Niger, quel avenir pour les éleveurs sahéliens ? Genève, Suisse, Bureau international du travail.

56. VIAS FRANCK S.G., BONFOH B., DIARRA A., NAFERI A., FAYE B., 2003. Dairy farms around Niamey: characteristics, productivity, marketing and milk quality. Revue Etud. Rech. sahél., 8-9: 159-165.

57. WILLIAMS T.O., TURNER M.D., 2002. Livestock market dynamics and local vulnerabilities in the Sahel. World Dev., 30: 83-705.

58. WYMANN M.N., 2005. Calf mortality and parasitism in periurban livestock production in Mali. PhD Diss., University of Basel, Switzerland, 227 p.

59. WYMANN M.N., BONFOH B., SCHELLING E., BENGALY S., TEMBELY S., MARCEL T., ZINSSTAG J., 2006. Calf mortality rate and causes of death under different herd management systems in peri-urban Bamako, Mali. Livest. Sci., 100: 169-178.

60. WYMANN M.N., BONFOH B., TRAORE K., TEMBELY S, ZINSSTAG J., 2007. Species diversity and acquisition of gastrointestinal parasites in calves aged 0-13 months in periurban livestock production in Mali. Vet. Parasitol., 143: 67-73.

Accepté le 12.03.2010

\section{Resumen}

Bonfoh B., Fokou G., Ould Taleb M., Fané A., Woirin D., Laimaibao N., Zinsstag J. Dinámicas de los sistemas de producción lechera, riesgos y transformaciones socio económicas en Mali

Como respuesta a la siempre creciente demanda urbana de leche y a las políticas de desarrollo del sector, la meseta lechera situada en la periferia de Bamako a sufrido importantes cambios desde la independencia. Las políticas industriales de los años 1970-80 han tenido impactos sociales importantes. Han sobretodo conducido al desarrollo de importaciones de leche en polvo y al salario de los pastores. Las políticas liberales que siguieron han más bien impulsado el desarrollo de pequeñas unidades de transformación y la intensificación de la cría pastoril. Pero en ausencia de control, estas políticas han también contribuido a importantes mezclas genéticas y al surgimiento de zoonosis y de riesgos de infección, peligrosos para la salud pública. Un enfoque integral, relacionando la adaptación de las técnicas de producción y el acceso a los mercados y los servicios, permitiría, con el apoyo de las políticas y en concertación con los actores, atenuar los riesgos de estas innovaciones y mejorar los medios de subsistencia de todos los involucrados.

Palabras clave: Producción lechera - Desarrollo agrícola Calidad - Salud - Malí. 